\title{
Palatal Presentation of Para-Pharyngeal Mass
}

\author{
$1 *$ Md. Ashraful Islam MBBS, FCPS, FICS, FRCS (Glasgow) \\ *Corresponding author \\ Professor and Head, Otolaryngology-Head \& Neck Surgery \\ Bangladesh Medical College Hospital
}

\author{
${ }^{2}$ Tareq Mohammad MBBS, FCPS \\ Assistant Registrar ENT \\ Bangladesh Medical College Hospital \\ ${ }^{4}$ Nazmul Hossain Chowdhury MBBS, FCPS
Assistant Professor, ENT
Bangladesh Medical College Hospital \\ ${ }^{6}$ Farid Uddin Milki, MBBS, DLO \\ Consultant ENT \\ Bangladesh Medical College Hospital
}

${ }^{3}$ ASM Lutfur Rahman MBBS, MCPS, DLO, MRCPS
(Glasgow) Resident Surgeon
Bangladesh Medical College Hospital

${ }^{5}$ Saif Rahman Khan MBBS
Assistant registrar, ENT
National Institute of ENT

${ }^{7}$ Towsif Bin Mamoon, MBBS

Assistant Registrar, ENT

Bangladesh Medical College Hospital

\begin{abstract}
Para-pharyngeal space tumors showed a varied presentation and through a great challenge to the surgeon in respect to pre-operative evaluation as well as surgical approach. Para-pharyngeal space (PPS) is an inverted cone-shaped potential space that extends from the hyoid bone to the base of the skull.It is filled with fat and areolar tissue lie laterally on either sides of pharynx; and is bounded and subdivided by various condensations of fascia ${ }^{1}$. Tumors, although rare less than $1 \%$ of head neck neoplasm can arise from any structures of this space. Mostly they are benign70-80\% and $20-30 \%$ are malignant. Salivary gland tumor, particularly deep lobe parotid constitutesmore than $80 \%{ }^{2,3}$. Imaging studies are invaluable to define the site of origin, extension, relationship to the great vessels of the neck and other neurovascular structures ${ }^{4}$. Surgery is the mainstay of treatment. Several approaches have been discussed in the text books. We present a case of a huge parapharyngeal space tumor that was excised completely through trans-cervical approach without any morbidly.
\end{abstract}

Keywords: Para-pharyngeal Space, Deep Lobe of Parotid, Trans-cervical Approach.

\section{INTRODUCTION}

Patients with tumors of the PPS most commonly present either in a neck or oropharyngeal mass that does not cause symptoms. It is important to have a clear anatomical picture in the surgeon's mind and heart to deal with the PPS lesions. PPS is an inverted pyramid shaped space, its base lies at the skull base and apex points inferiorly to the styloglossus muscle at the level of the angle of the mandible on each side of the neck that extends up to the greater cornu of hyoid bone. The fascia from the styloid process to the tensor velipalatini divides the parapharyngeal space into an anterior (prestyloid) compartment and a posterior (poststyloid) compartment.

Pre-styloid compartment houses the deep lobe parotid, ectopic salivary glands, a small branch of the trigeminal nerve, the ascending pharyngeal artery, the pharyngeal venous plexus, and lymph nodes. The post-styloid compartment contains mainly adipose tissue, internal carotid artery (ICA) and internal jugular vein (IJV), cranial nerves IX-XII, the cervical sympathetic trunk, and lymph nodes.

Most PPS tumor is benign, comprising $70-80 \%$. Mostly they are salivary gland origin accounting 60-70\% and usually arise from the deep lobe of parotid gland, ectopic salivary gland or in minor salivary glands of the lateral pharyngeal wall. The most common prestyloid PPS lesion is "pleomorphic adenoma" represents $80-90 \%$ of salivary neoplasms in the $\mathrm{PPS}^{2,3,4}$.Many other lesion of this compartment belongs to neurogenic tumors especially Schwannomas, paragangliomas, and lymphoreticular lesions.

In most of the cases the tumor remains asymptomatic and becomes an incidental finding.It can present with dysphagia, sore throat, feeling of lump in the throat, neck swelling etc. In this article we present a case of 15 years old girl reported with a huge swelling in the oral cavity.

\section{CASE REPORT}

A 15 years old beautiful girl presented with a gradually progressive painless swelling in the throat and change in quality of speech for one-year. According to her statement, she was reasonably well 4 years back then she started feeling pricking senation in the throat. While she the girl tried to see her throat wth a mirror, she incidentally 
discovered a huge bulging in her right of palate.Inspite of the fact, she did not develop anysignificant symptoms for over 3 years and had not disclosed to her parents. Since then she experienced little change ofher voice and subseqently she also noticed a swelling in ipsilateral upper neck.

On examination of the oral cvity and throat, there was a smooth bulge of right half of soft palate(Figure 1) and right lateral pharyngeal wall with medial displacement of right tonsil and left lateral displacement of uvula. Neck examination revealed a diffuse swelling in the level $1 \mathrm{~b}$,

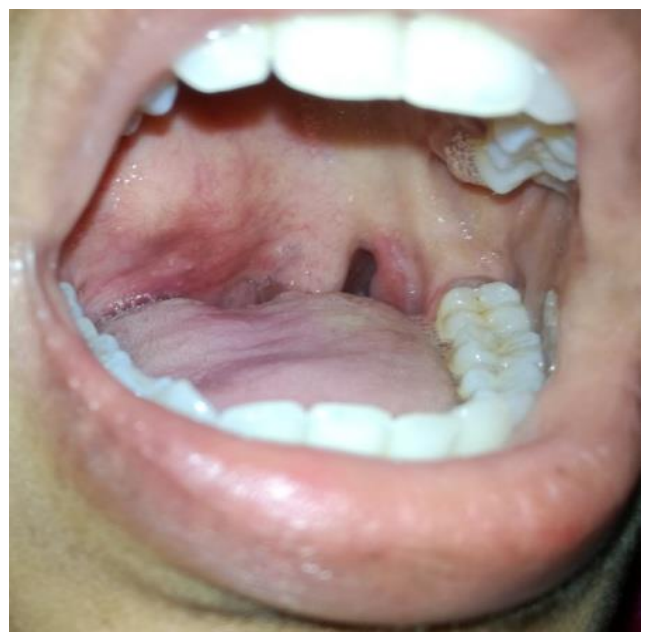

a
Peroral palpation the mass was firm, non tender and bosselated surface on the palate extended to nasopharynx, with smooth palpable swelling in the floor of mouth, . Postnasal examination showed extension of the swelling into the nasopharynx and indirect laryngoscopy revealed the lower limit of swelling at the level of vallecula. Neck palation revealed a non tender diffuse swelling, overlying structures remained free and bimanual palpable. There was no significant lymph node enlargement in the neck. Her FNAC suggested it as pleomorphic adenoma.

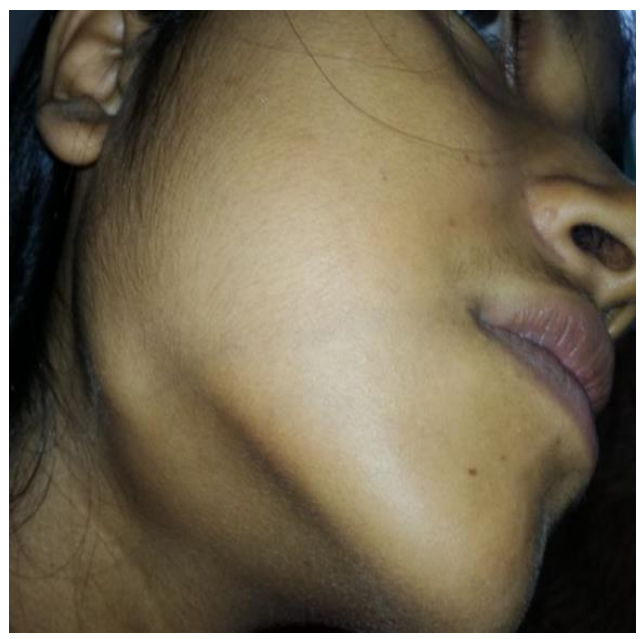

b

Fig 1. a,b:- Bulged right side of soft palate (a) and submandibular swelling (b)

On the basis of history, physical examination and FNAC finding, it was diagnosed as a case of right parapharyngeal mass.MRI of oral cavity and neck was done and showed a well circumscribed lobulated soft tissue mass measuring $6.5 \times 4.5 \times 6.2$ $\mathrm{cm}$ in the right parapharyngeal space, extending from the base of skull to vallecula, crossing the midline, and compressing the ICA and IJV (Figure $2 \mathrm{a}, \mathrm{b}$ ).

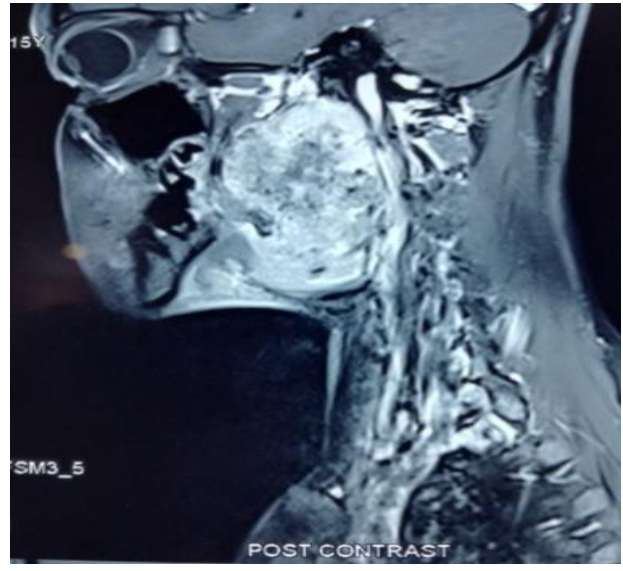

a

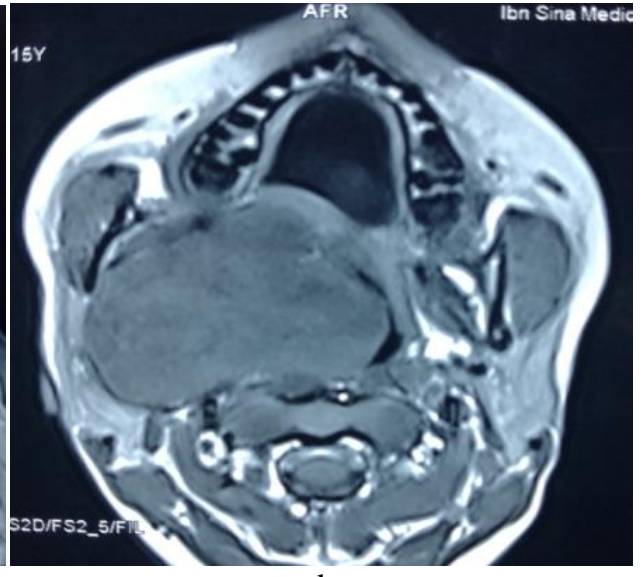

$\mathrm{b}$

Fig 2 a,b: MRI Oral cavity including neck, showing the extension of tumor 


\section{SURGICAL PROCEDURE}

Through a submandibular transcervical approach, the parapharyngeal space was accessed; a tunnel made between the submandibular gland and vascular triangle after lateral mobilization of SCM.

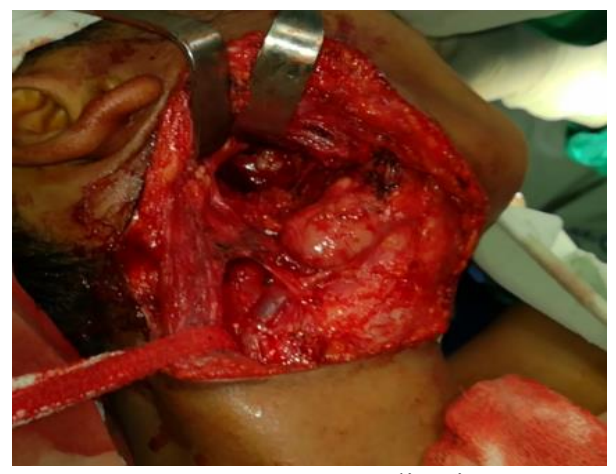

a. SCM Lateralization

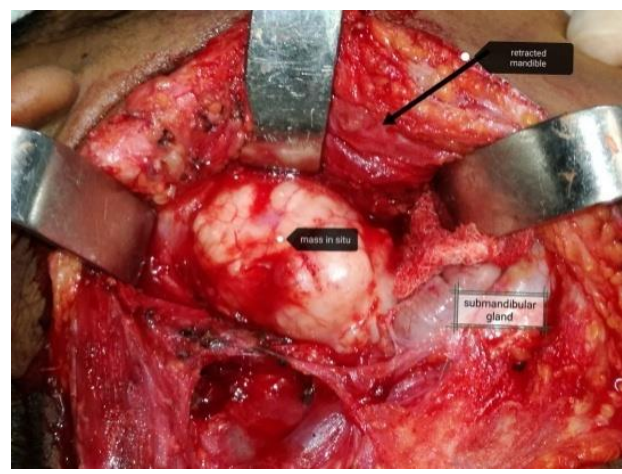

b. Exposure of field with retraction of mandible

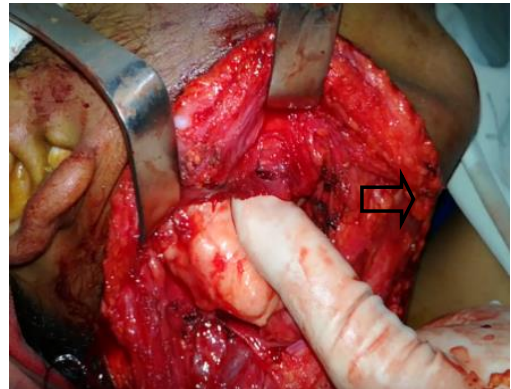

c.

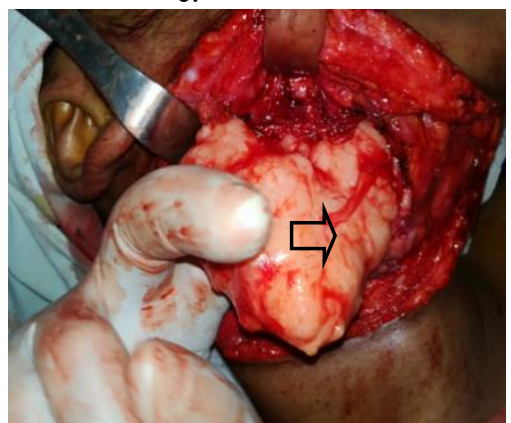

f.

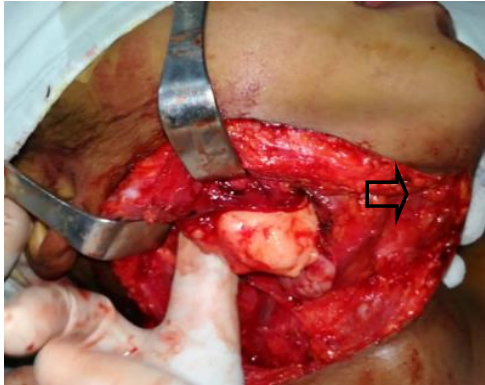

d.

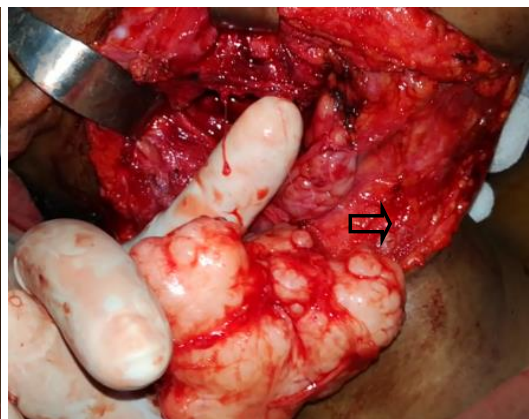

g.

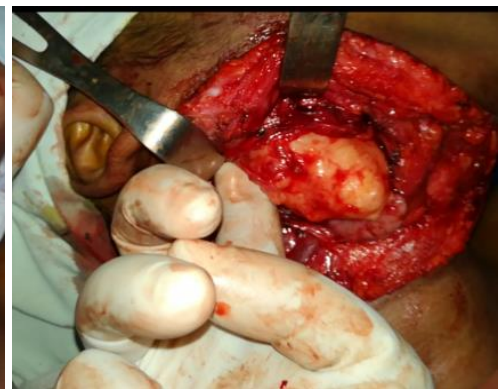

e.

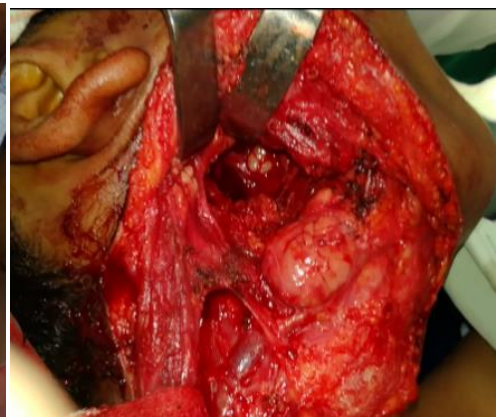

h.

Fig 3(a-h): Step of dissection

The mass first separated from the vascular triangle and mobilized from the infratemporal fossa, nasopharynx, and oropharynx and completely extirpated from the adjuscent areas within only 10 minutes with a very minimum blood loss (approx $<100 \mathrm{ml}$ ). The mobilization and separation mostly controlled by the finger(Figure 3 ) (Total operation time from incision to closer was less than 1 hour). The mass measured $6 \times 5.5 \times 4 \mathrm{~cm}$.(Figure 4 ).

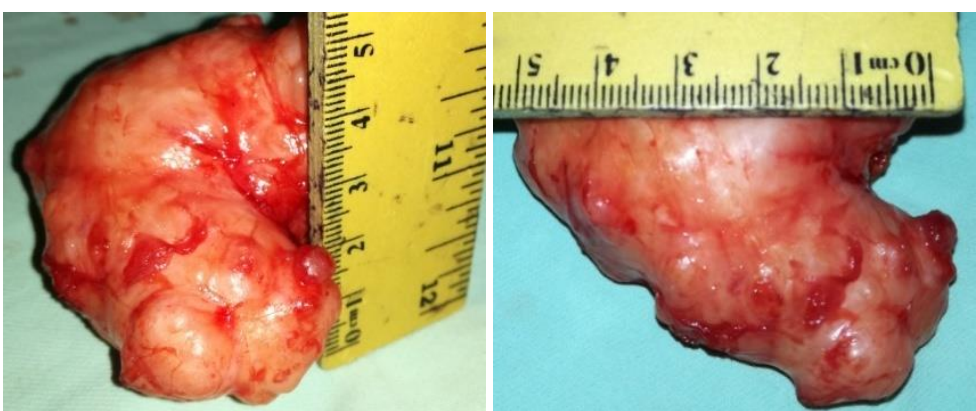

a. b.

Figure 4a,b: Size of lesion after excision 
Histopathologically showed features of a pleomorphic adenoma, composed of epithelial and myoepithelial cells diposed in chondro-myxoid background.

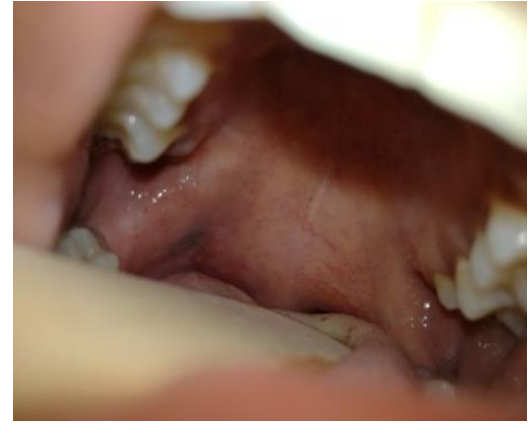

a.

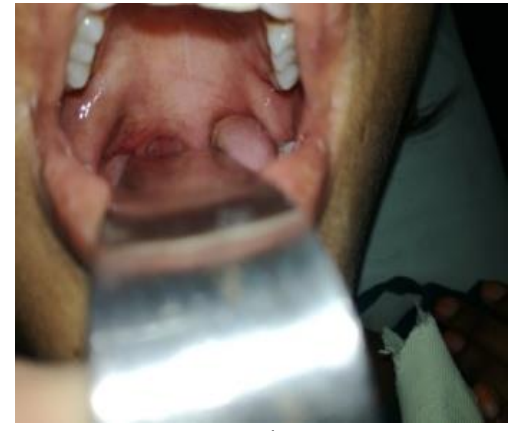

b.

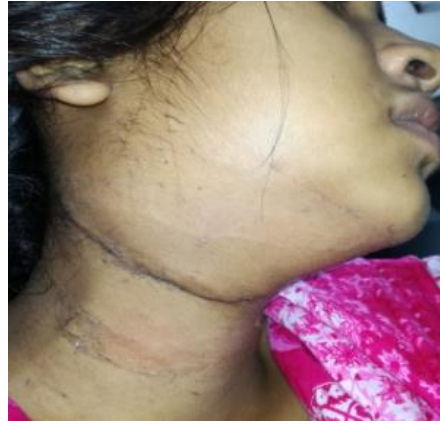

c.

Figure 5 a,b,c: post operative picture.

\section{DISCUSSION}

Para-pharyngeal space tumors showed a varied presentation and through a great challenge to the surgeon in respect to pre-operative evaluation as well as surgical approach. Patients with tumors of the PPS most commonly present either in a neck or oropharyngeal mass that does not cause symptoms. Para-pharyngeal space is a potential space of head and neck that may become involved by various pathological entities such as infections, inflammatory, and neoplastic. Tumors of this PPS represent less than $1 \%$ of all head and neck tumours ${ }^{6,7,8}$. Both benign and malignant tumors may arise from any structure contained within the PPS where 70-80\% appears belong to benign and 20-30\% to malignant. Of the benign tumors pleomorphic adenoma is the commonest ${ }^{9}$. The most common site of a pleomorphic adenoma of the minor salivary gland is on the palate followed by lip, buccal mucosa, and floor of mouth ${ }^{10}$.Pleomorphic adenoma of PPS can arise from deep lobe of parotid or ectopic salivary glands ${ }^{[11-13]}$ which was correlated in our case.

The presentation is variable, most of these tumorsremain asymptomatic for long time but may present with dysphagia, voice change, sore throat, otalgia, lower cranial palsy etc. Classically the benign lesions are painless, firm and mobile. A lateral pharyngeal swelling or a palatal bulging along with medial tonsillar and lateral uvular displacement is diagnostic of a para-pharyngeal mass with or without extension to retromandibulartrigone and the submandibular triangle. In most of the cased the submandibular swelling remains bimanually palpable $e^{6,13,14}$ that was aptly found in this girl.

The diagnostic workup algorithm begins with hematologic and serologic tests. Fine needle aspiration cytology (FNAC), CT and/or MRI studies should be the part of the preoperative diagnosis to determine the extent of disease, local spread, and even the type of tumor. Preoperative FNAC and was carried out and was diagnostic in our case.
Cross sectional imaging is mandatory to see the extension, character and site of origin of the lesion. MRI is superior then CT as it helps to identify the site of origin. A CT scan is cost effective and with contrast ascertains the vascularity of tumors and their relationship to adjacent neurovascular structures. The presence or absence of fat planes between the tumor and adjacent structures and the direction in which the tumor displaces the fat plane helps to determine whether the tumor is located in the pre-styloid or post-styloid compartment and whether the site of origin is the parotid gland ${ }^{5,8,15}$. In addition, a CT scan provides a precise definition of the skeletal framework, demonstrating bone remodeling, expansion, or destruction.

The mainstay of treatment of tumors of the parapharyngeal space is surgical extirpation.though choice of surgical approach is challenging due to its location with cranial nerves, great vessels sympathetic chain ${ }^{15}$. Complete excision of the tumor is recommended to corroborate the diagnosis suggested by the clinical examination and imaging. Several approaches has been recommended likeTransoral, Transcervico-Submaxillary, Transcervical, Transmandibular, Transparotid, Infratemporal Fossa approach $^{16}$ etc. However different approaches have individual co-morbidity. In an encapsulated tumor of PPS, trans-cervical approach remains beneficial as mandibular splitting can be avoided though there is chance of marginal mandibular nerve injury, but can easily be avoided. In our case we used the submandibular trans-cervical approach and the mandible was retracted superiorly for better exposure and mass can be removed by blunt dissection.

\section{CONCLUSION}

It is challenging for a surgeon to choice the best approach to a para-pharyngeal space tumorwithout morbidity Cross sectional imaging is best to diagnose and assess extension of the tumor; and it also aids to choose the surgical approach. A large encapsulated tumor can come easily through a trans-cervical approach without mandibular splitting.

Disclosure: There is no financial relation or funding and no conflict of interest. 


\section{REFERENCES}

[1]. Andrew S jones, Scott-Browns Otolaryngology, Head and Neck surgery, $7^{\text {th }}$ edition 2522

[2]. Johnson AT, Maran AG, Extra cranial tumors of the infratemporal fossa. Journal of laryngology and otology. 1982: 96: 1017-1026.

[3]. Ricardo L. Carrau, Jonas T. Johnson, Eugene N. Myers. Management of tumors of the parapharyngeal space. Cancer network, Head neck cancer, 1997.v-11.

[4]. Kassel EE, Parapharyngeal and deep lobe parotid tumors, Journal of otolaryngology. Supplement. 1982: 12: $25-35$.

[5]. Curtin HD: Separation of the masticator space from the space. Radiology 1987.163:192-204.

[6]. Work W, Hybels RL: A study of tumors of the parapharyngeal Laryngoscope 1974.84:1748-1755.

[7]. .Batsakis JG, Nelge N: Parapharyngeal and retropharyngeal space diseases. Ann Otol Rhinol Laryngol 1989.98:320-321.

[8]. Carrau RL, Myers EN, Johnson JT: Management of tumors arising in the parapharyngeal space. Laryngoscope 1990.100:583-589.

[9]. B. T. Verghese, P. Sebastian, E. K. Abraham, and A. A. Mathews, "Case report: pleomorfic adenoma of minor salivary gland in the parapharengeal space," World Journal of Surgical Oncology, vol. 1, no. 2, 2003.

[10]. B. Sergi, A. Limongelli, E. Scarano, A. R. Fetoni, and G. Paludetti, "Giant deep lobe parotid gland pleomorphic adenoma involving the parapharyngeal space. Report of three cases and review of the diagnostic and therapeutic approaches," Acta Otorhinolaryngologica Italica, vol. 28 , no. 5, pp. 261265, 2008.

[11]. N. Morita, K. Miyata, T. Sakamoto, and T. Wada, "Pleomorphic adenoma in the parapharyngeal space: report of three cases," Journal of Oral and Maxillofacial Surgery, vol. 53, no. 5, pp. 605-610, 1995.

[12]. P. W. Work and G. A. Gates, "Tumours of parapharyngeal space," Otolaryngologic Clinics of North America, pp. 479-514, 1969.

[13]. Carrau RL, Myers EN, Johnson JT. Management of tumours arising in the parapharyngeal space. Laryngoscope. 1990;100:583-589

[14]. Som PM, Biller HF, Lawson W, et al: Parapharyngeal space masses: An updated protocol based upon 104 cases. Radiology 153:149-156, 1988.

[15]. K. D. Olsen, "Tumors and surgery of the parapharyngeal space," Laryngoscope, vol. 104, no. 5, pp. 1-28, 1994.

[16]. F. Bozza, M. G. Vigili, P. Ruscito, A. Marzetti, and F. Marzettt, "Surgical management of parapharyngeal space tumours: results of 10-year follow-up," Acta Otorhinolaryngologica Italica, vol. 29, no. 1, pp. 10$15,2009$.
[17]. Istemiahn A, tugba K, Murad M, Mehmet S, and Evrim Ö.Pleomorphic Adenomas of the Parapharyngeal Space, Volume 2014 Article ID 168401, 4 pages, 2014

[18]. Nora Siupsinskiene, Irina Arechvo, RimanteLapinskaite, EvaldasPadervinskis, Si lvijaRyskiene, and SauliusVaitkus. A rare case of Large Schwannomaof parapharyngeal space. Case Reports in Otolaryngology / 2018. Volume 2018 |Article ID 9870937 